BNL-114172-2017-JA

\title{
Perspectives on in situ electron microscopy
}

\author{
H. Zheng, Y. Zhu
}

Submitted to Ultramicroscopy

September 2017

\begin{abstract}
Condensed Matter Physics and Materials Science Department
Brookhaven National Laboratory
\end{abstract}

\author{
U.S. Department of Energy \\ USDOE Office of Science (SC), \\ Basic Energy Sciences (BES) (SC-22)
}

Notice: This manuscript has been authored by employees of Brookhaven Science Associates, LLC under Contract No. DE- SC0012704 with the U.S. Department of Energy. The publisher by accepting the manuscript for publication acknowledges that the United States Government retains a non-exclusive, paid-up, irrevocable, world-wide license to publish or reproduce the published form of this manuscript, or allow others to do so, for United States Government purposes. 


\section{DISCLAIMER}

This report was prepared as an account of work sponsored by an agency of the United States Government. Neither the United States Government nor any agency thereof, nor any of their employees, nor any of their contractors, subcontractors, or their employees, makes any warranty, express or implied, or assumes any legal liability or responsibility for the accuracy, completeness, or any third party's use or the results of such use of any information, apparatus, product, or process disclosed, or represents that its use would not infringe privately owned rights. Reference herein to any specific commercial product, process, or service by trade name, trademark, manufacturer, or otherwise, does not necessarily constitute or imply its endorsement, recommendation, or favoring by the United States Government or any agency thereof or its contractors or subcontractors. The views and opinions of authors expressed herein do not necessarily state or reflect those of the United States Government or any agency thereof. 


\title{
Perspectives on In-situ Electron Microscopy
}

\author{
Haimei Zheng ${ }^{1,2}$ and Yimei Zhu ${ }^{3,4}$ \\ ${ }^{1}$ Materials Sciences Division, Lawrence Berkeley National Laboratory, Berkeley, CA 94720, \\ USA \\ ${ }^{2}$ Department of Materials Science and Engineering, University of California, Berkeley, CA \\ 94720, USA \\ ${ }^{3}$ Department of Condensed Matter Physics and Materials Science, Brookhaven National \\ Laboratory, Upton, NY 11973, USA \\ ${ }^{4}$ Department of Physics and Astronomy, Stony Brook University, Stony Brook, NY 11790, USA \\ Correspondence to hmzheng@lbl.gov or zhu@bnl.gov
}

\section{Introduction}

In situ electron microscopy has been identified as one of the major future directions of electron microscopy (BES workshop report[1]). It allows us to retrieve information on materials' structural dynamics under applied excitation or during chemical reactions, discover transient states related to materials behavior and correlate materials structure to their functionality[2-5]. There are many fascinating research topics using in situ transmission electron microscopy (TEM) owing to the development in situ methods, ranging from liquid and gas environment TEM[6-8], to pump-probe ultrafast microscopy[9-11], nanomechanics[12, 13] and ferroelectric domain switching[14, 15]. With the recent advances in electron microscopy including aberration corrected optics, sample environment control, sample stage, fast and sensitive data acquisition, in situ electron microscopy has become increasingly powerful and popular. For example, the aberration corrected TEM has enabled spatial resolution in the sub- $\AA$ range and energy resolution in the sub-eV range a routine. In situ measurements with aberration corrected high resolution imaging, electron energy loss spectroscopy (EELS), electron energy dispersive x-ray spectroscopy (EDS) and highly sensitive fast electron detection allow many discoveries on materials dynamic processes at the atomic level that have not been previously possible. Thus, it will bring more impact in a broad areas of research from materials science to physics, chemistry and biology.

In this article, a few recent in situ TEM work in the area of materials chemistry, such as colloidal nanoparticle growth and electrochemical processes using liquid environmental cells, and in solid state physics, such as topological vortices in ferroelectric and ferromagnetic 
materials, ferroelectric or ferromagnetic domain switching are highlighted. An outlook into future challenges and opportunities in in situ TEM including the impact of aberration corrected TEM are provided.

The development of liquid cells has opened many opportunities for the study of materials dynamic processes in liquids with TEM, such as, nucleation and growth, self-assembly of nanomaterials, electrochemical processes, biological materials in physiological conditions, etc. In the past a few years, publications on the topic of liquid cell TEM study have increased rapidly.[7] Here, the observed nanoparticle growth in a liquid cell with high resolution imaging and fast electron detection, the lithiation/delithation of $\mathrm{MoS}_{2}$ nanoflakes in an electrochemical cell with EDS or other spectroscopic measurements show the growing impact of in situ electron microscopy in materials chemistry by characterization of dynamic processes of materials with high spatial and temporal resolution.

In the second part of this paper, a couple of examples on in situ electron microscopy of ferroelectric or ferromagnetic vortexes and domain switching are provided. The 2016 Nobel Prize in Physics awarded to three scientists for their discovery of topological phase transitions and topological phases of matter generated great excitement. Topological structures, emerging near spontaneous symmetry-breaking transitions, are ubiquitously observed in wide branches of science.[16-21] In condensed matter, topological defects can be promising candidates for information storage technology. Skyrmions, multiferroic vortices, domain walls, dislocations, and disclinations are examples, where emergent properties and behaviors have been reported.[22-27] These topological defects are invariant under continuous deformations or perturbations, and thus said to be protected by topology. They often are observable and play important roles in phase transition. Their fascinating underlying physics responsible for striking geometric patterns can be found in order parameter space.[28] In situ TEM is a unique approach to topological structures, where the aberration corrected electron microscopy plays a major role.

\section{In situ study of materials dynamics in liquids}

Most liquids including water and other organic solvent have a high vapor pressure, thus they are incompatible with the high vacuum environment of a transmission electron microscope. To separate those liquids from the vacuum environment, a sealed liquid cell is required. A liquid cell needs a thin imaging window allowing the electron beam to go through while being able to 
survive in the high vacuum. The concept of windowed TEM cell was discussed in the earliest days of electron microscopy.[29] From the early attempt of imaging wet samples using sandwiched thin aluminum foils in 1930s'[30] to the liquid cells nowadays with thin SiNx/Si membrane or graphene or functional liquid cell with applied electric bias, tremendous progress has been made and breakthrough discoveries have been achieved.

The windowed self contained liquid cells with ultra thin SiNx membrane[8] (as thin as $10 \mathrm{~nm}$ ) or graphene offer superb image resolution. SiNx membranes are mechanically strong, inert, with low imaging contrast and excellent as a support for electrode patterning. Various types of patterned electrodes (e.g., $\mathrm{Au}, \mathrm{Ti}, \mathrm{Al}, \mathrm{Pt}$, graphene, etc.) can be made into an electrochemical liquid cell with SiNx membranes. It is also possible to flow liquids between the two silicon nitride membranes of a liquid cell using external tubing and a syringe pump, which has been widely used in a commercial liquid cell stage and is attractive for the study of reactions with instant mixing of liquids.

Liquid cell TEM has been used for the exploration of new frontiers for materials synthesis, electrochemistry, catalysis, fluid physics, biological materials in aqueous environments and many other areas of research[3]. Compared to many other in situ approaches, such as in situ optical spectroscopy,[31] x-ray spectroscopic methods,[32-36] atomic force microscopy (AFM) and scanning tunneling microscopy (STM),[37-39] in situ TEM has its unique advantages allowing directly observation of materials transformation dynamics in liquids with high spatial and temporal resolution. The major criticisms come from the electron beam radiation damage and the challenges in controlling of local environment, reactant mixing, etc. during reactions, which will be discussed in the last section of this article.

\section{Colloidal nanocrystal growth}

Crystallization process is complex involving the arrangement of thousands of atoms or molecules near the surface, and it is further complicated by the interactions between atoms and influences from the surroundings. With modifications of the temperature, precursor and surfactants, it is possible to make nanocrystals with different shape and structure. Liquid cell TEM is a unique and effective method for the study of nanoparticle growth mechanisms revealing the role of different factors in controlling structure and morphology of nanoparticles. 
There have been many reports on nanocrystal growth mechanisms using liquid cell TEM. Here, we show a series of studies on nanoparticle shape evolution and highlight the significant progress that has been made due to the combination of liquid cell development, fast electron detection and advanced electron microscopy. In order to achieve monodisperse nanoparticles, it is often considered that coalescence between nanoparticles should be avoided. Real time observation indicates that two types of growth, either by monomer attachment or by coalescence, lead to the same particle size (Fig. 1a).[8] The fascinating growth by nanoparticle attachment has also been observed in Pt-Fe nanowire formation[40]. The interactions between Pt-Fe nanoparticles result in nanoparticle end-to-end attachment into a chain (Fig. 1b). Further systematic studies have been carried out to show that surfactants can drastically influence morphology of a nanoparticles, modify the growth rate of certain facets, inhibit nanoparticle aggregation, and prevent coalescence.

Wulff construction[41, 42] has been used to predict the equilibrium shape of nanocrystals, where it states that the growth rate of certain facet is proportional to the surface free energy. For the growth of nanocrystals the high-energy facet grows at a higher rate than the low-energy facets, therefore, the fast growing facets will eventually disappear resulting in a nanocrystal terminated with low energy facets.[43, 44] It is assumed that the commonly used surfactants modify the energy of specific facets through preferential adsorption, influencing the relative growth rate of different facets thus the shape of a nanocrystal[45, 46]. However, in situ observation of Pt nanocube formation liquid cell TEM shows drastic deviation from the Wulff construction prediction.[47] As shown in Fig. 1c\&d, we found the growth rates of all low index facets, $\{100\},\{110\}$ and $\{111\}$, are similar until the $\{100\}$ facets stop growing. The $\{110\}$ facets will continue to grow until they reach a limit, at which point they form an edge of a nanocube. The continued growth of $\{111\}$ facets fills the corners of the cube. Such atomic facet development in platinum nanocube growth suggests the surface energy minimization rules breaks down at the nanoscale. Density functional theory (DFT) calculation reveals that the drastic differences of ligands mobility on different facets, i.e., ligands move several orders of magnitude slower on $\{100\}$ than $\{111\}$ facets retarded the growth of $\{100\}\}$ facets. Therefore, a selective facet arrested shape control mechanisms is proposed. The improved liquid cells with thinner SiNx membranes and a fast electron detector with high detection quantum efficiency (K2 IS camera) are critical for achieving high spatial resolution. 
Park et al. used an aberration-corrected TEM and direct electron detector to resolve the growth and 3D atomic structures of Pt nanocrystals in a graphene liquid cell[48, 49]. Graphene liquid cells offer unprecedented resolution (Fig. 2). Observation reveals that coalescence is siteselective and the $\{111\}$ facets with lowest surface energy or lowest ligand coverage are preferred for nanoparticle attachment[49].

\section{$\underline{\text { Electrochemical process induced materials degradation }}$}

An electrochemical process can be visualized with an electrochemical liquid cell where electrodes are built inside the liquid cell. Electrochemical lithiation of Au electrode, dendritic growth of lead, lithium or other structures and the subsequent stripping of electrodeposited materials have been observed using an electrochemical liquid cell. In addition, the formation of SEI layer and characterized the structure of SEI in situ (i.e., LiF nanoparticles uniformly distributed within the SEI) with advanced electron microscopy techniques have also been achieved. Recently, with a nanobattery cell with commercial electrolyte $\mathrm{LiPF}_{6} / \mathrm{EC} / \mathrm{DEC}$ for batteries and Li metal on one electrode as counter (and reference) electrode, structure changes of $\mathrm{MoS}_{2}$ nanoflakes after lithiation/delithiation have been observed.

The fabricated nanobattery cell for TEM offers the opportunity to study the reaction mechanisms of $\mathrm{MoS}_{2}$ during battery operation. Further characterization after the reaction was stopped in the liquid cell, for instance, elemental analysis using EDS and structural identification with nanobeam diffraction provide valuable complementary information.

Layered $\mathrm{MoS}_{2}$ is an attractive electrode material for lithium ion batteries.[50]'[51] When lithium ions are inserted between the layers of $\mathrm{MoS}_{2}$, the $\mathrm{MoS}_{2}$ structure can be exfoliated by ultrasonication of lithiated $\mathrm{MoS}_{2}$ in $\mathrm{H}_{2} \mathrm{O}$.[52, 53] Electrochemical degradation may be induced by the active material loss when $\mathrm{MoS}_{2}$ is used as the cathode material.[54] In situ observation of lithiation and delithiation of $\mathrm{MoS}_{2}$ as the electrode material provides critical insight on the degradation mechanisms of this material for battery applications.

In situ studies show dissolution and lithiation induced deformation of $\mathrm{MoS}_{2}$ during charge in the voltage range of $1.8 \sim 1.2 \mathrm{~V}$. Irreversible decomposition near $1.1 \mathrm{~V}$ was observed, where $\mathrm{MoS}_{2}$ nanosheets broke down into $5 \sim 10 \mathrm{~nm} \mathrm{MoS}$ nanoparticles as confirmed by nanobeam diffraction experiments (Fig. 3). Some $\mathrm{MoS}_{2}$ nanosheets decompose upon lithiation while the 
other remain and experience structural changes, i.e., swelling or deformation. The variation of the $\mathrm{MoS}_{2}$ nanosheets during lithiation may result from the $\mathrm{MoS}_{2}$ thickness difference or inhomogeneity during lithiation. Out in-situ study of $\mathrm{MoS}_{2}$ decomposition during lithiation in battery operation provides insights on improving the battery design and applications of transition metal dichalcogenides (TMDCs) and layered structured materials in energy devices.

\section{In situ study of topological vortices in ferroelectric and ferromagnetic materials}

Understanding topological structures is crucial to the prediction of behavior and functionalities emerging from the topological defects, such as skyrmions, multiferroic vortices, domain walls, dislocations, and disclinations.[55] Thus, it is important to characterize domaindefect interactions and their dynamics, as well as to directly link local atomic-displacement to polarization.[22, 56-60] Lorentz microscopy has been widely used to study the skyrmions (ref. Pollard, S.D., Garlow, J.A., Yu, J., Wang, Z., Zhu, Y., Yang, H., “Observation of stable Néel skyrmions in Co/Pd multilayers with Lorentz TEM", Nature Communication, in press), the magnetic switching

and domain configuration evolution. The dynamical process of generation and annihilation of the magnetic biskyrmions have been observed by in situ TEM[59]. As an alternative approach, a dedicated magnetizing stage can be used to generate a magnetic field at the specimen area by adding Helmholtz coils on each side of the specimen for the study of magnetic field induced motion of magnetic domain walls [61, 62]. Another possible design is bring the piezo-driven sharp needle made of a permanent magnet close to the specimen[63, 64]. To draw the relationship between magnetic structures and temperature, a thermal element also need be integrate with the holder. Meanwhile, a new design capable of applying GHz resonance electric current and pulsed excitations in situ was developed to measure the non-adiabatic spin torque effect[65], and to map strongly coupled coaxial vortex motion in the dipolar- and indirect exchange-coupled regimes.[66]

\section{Ferroelectric vortices}

In rare-earth hexagonal manganites $\mathrm{RMnO}_{3}(\mathrm{R}=\mathrm{Er}, \mathrm{Y})$, vortices and antivortices are topological defects. At high temperature the crystal structure adopts centrosymmetric $\mathrm{P}_{3} / \mathrm{mmc}$. A structural transition occurs at Tc, which lowers the symmetry to $P 6_{3} \mathrm{Cm}$ mainly by the condensation of the $\mathrm{K}_{3}$ phonon mode. This process leads to the trimerized tilting of $\mathrm{MnO}_{5}$ bipyramids and corrugation of intercalated R layers while maintaining the six-fold symmetry 
with six crystallographically preferred domains denoted as $\alpha+, \beta-, \gamma^{+}, \alpha-, \beta+, \gamma-$ in sequence around the core[67]. These six-fold vortices are topologically protected and extremely stable under thermal perturbation and external biasing[68-70]. In-situ biasing in TEM is the most effective approach to study individual ferroelectric vortex and antivortex formation and inhalation and their dynamic switching behavior. Fig. 4a-c give an example of hexagonal $\mathrm{ErMnO}_{3}$, a geometric ferroelectric since its spontaneous polarization is induced by the structural trimerization of Mn ions and the buckling of Er ions due to mismatch in ionic size between the R-ions and the Mn ions, as shown in Fig. 4c. The symmetry-breaking phase transition of the Mn trimerization engenders two possible directions of polarization along the c-axis (“+” being parallel to the c-axis, and “-“ being antiparallel to it ), and three antiphases ( $\alpha, \beta$, and $\gamma)$, totaling six distinctive domains $(\alpha+, \alpha-, \beta+, \beta-, \gamma+, \gamma-)$ [67]. The walls of the trimerization domain, interlocked with ferroelectric domain walls, emerged from topological defects as vortex and antivortex (Fig. 4a) with opposite winding orders. The vortices are quite stable. Even in the presence of severe discontinuities in electric polarization around the core, the formation of these topological defects are observed when the crystal is exposed to temperatures above Tc. There are two types ferroelectric domain walls, type-A and type-B, with lattice shift of $1 / 3[120]$ and 2/3[120], across the domain wall, respectively. By measuring the area of polarized domains insitu with applied electric field we can correlate nanoscale polarization to microscale hysteresis property (polarization vs electric field loop) of the sample. For example, when we vary the field from $-150 \mathrm{kV} / \mathrm{cm}$ (as shown in Fig. 4b) to $150 \mathrm{kV} / \mathrm{cm}$ we can direct observe the ferroelectric switching behavior: domains parallel to the field direction expand while those antiparallel to the field direction shrink. Nevertheless, the vortex cores are immobile. We note when two domains are paired they carry opposite charges, resulting in a strong attractive interaction. Fig. 4c shows the bound states carrying domains can be as narrow as only 1 unit-cell wide for oppositely charged paired walls, as marked on top left corner of figure. We attribute the stability of these narrow domains to the incommensurability of partial unit-cell-shifts across the paired walls that prevent the unification of the bound states, or pair-annihilating even with one unit-cell separation. It assures the strong short-range repulsive interaction for the bound states. These narrow domains are topologically protected due to the incommensurate sum of the partial unit-cell-shift vectors for each pair of walls, preventing domain annihilation resulting in incomplete polling, a feature that was not observed in stereotypical ferroelectrics, such as $\mathrm{PbTiO}_{3}$ and $\mathrm{BaTiO}_{3}[69,71]$. 
Six-fold vortex domains are most common in $\mathrm{RMnO}_{3}$, as they reflect the six crystallographic variants. Non-six-fold vortex domains are inconsistent with the crystal symmetry, thus do not expect to exist in hexagonal manganites. Nevertheless, 2-fold, 4-fold, and 8-fold vortex domains have been recently observed. An example for an eight-fold vortex structures using aberrationcorrected electron microscopy is shown in Fig. 4 (d), revealing startling detailed atomic configurations of crystallographically forbidden non-six-fold ferroelectric domains surrounding the vortex cores in hexagonal $\mathrm{YMnO}_{3}$. The vortex core is incorporated with two partial edge dislocations (PEDs), another important class of topological defects. The polarization directions of the ferroelectric domains can be distinguished by corrugated configurations of Y-ions, similar to 6-fold vortices. To map the associated strain field of the PEDs of the eight-fold vortex cores, we used geometric phase analyses (GPA)[72], and the strain filed of $\varepsilon_{\mathrm{xx}}$ (the $\mathrm{x}$ axis is defined as the [120] direction) around the PEDs constructed from the atomic images, as shown in the insets. Clearly, the non-uniform displacement field near the vortex centers (marked by the circles), especially near the PED cores, plays a significant role in altering the corrugated configuration of the vortex structure. The corresponding mesoscale dark-field images that possess dissimilar contrast for oppositely polarized ferroelectric domains due to the breaking of Friedel's law, are also included. Careful atomic image analysis suggests that the PED possesses a Burgers vector of 1/3[120]. To clarify the vortex-forming mechanism, Landau-theory based phenomenological model was used and the origin of the remarkable atomic arrangement and the intertwined relationship between the two types of topological defects, i.e. (anti)vortex cores and partial-edgedislocations (PEDs), as well as the roles of their displacement field, formation temperature and nucleation sites are revealed. All conceivable vortices in the system are topologically classified using homotopy group theory and their origins are identified. The unanticipated symmetry breaking was found at both mesoscale (domains) and atomic scale (vortex cores) due to the intertwining of two types of topological defects. Due to the interaction of PEDs with surrounding lattice, the initially topologically protected 6-fold (anti)vortex core structures can be transformed into other configurations. Thus, PED, depending on its characteristics, including Burgers vector, formation temperature and nucleation sites, can behave as a control knob for regulating vortex domain symmetry, whatever the methods that could introduce PEDs into this system. The ability to manipulate and control the ferroic orders in $\mathrm{RMnO}_{3}$ in correlation with spontaneous 
magnetization, electric polarization, and spontaneous strain may provide a platform for exploring emerging physical phenomena with novel applications via topological defects.

\section{Ferromagnetic vortices}

A ferromagnetic vortex is most commonly seen in a magnetic disc or square where in-plan spins circulate form a magnetic vortex at the center with an out-of-plan spin configuration (Fig.5a). Understanding switching behavior of ferromagnetic nanostructures will enable us to design better spintronic devices. In-situ magnetic imaging with Lorentz microscopy has been widely used to study topological defects and quasiparticles, including skyrmions and the evolution of spin structures under magnetic field or electric current. The dynamic switching process of generation and annihilation of spin configurations can be directly observed by applying magnetic field using a sample stage with build-in Helmholtz coils or a piezo-driven magnetic needle,[61, 64, 73] or by tilting the sample to utilize the magnetic field of the objective lens. A design capable of applying gigahertz resonance electric current and pulsed excitations in TEM was recently developed to measure spin vortex precession orbit and to understand the nonadiabatic spin torque effect.[65] Spin-transfer torque offers great promise for the development of spin-based devices. The effects of spin-transfer torques are typically analyzed in terms of adiabatic and non-adiabatic contributions. Currently a comprehensive interpretation of the non-adiabatic term remains elusive, with suggestions that it may arise from universal effects related to dissipation processes in spin dynamics, and other studies indicating a strong influence from the symmetry of magnetization gradients. The Lorentz microscopy with gigahertz-

excitations approach can achieve better than 5nm spatial resolution to map the orbit of a magnetic vortex core.

Fig. 5a shows a schematic of the spin vortex precession orbit measurement setup. The top corresponds to the sample assembly with its actual Lorentz image at the bottom. A high frequency ac signal is supplied externally through gold contact pads using waveguides to inject high-frequency current. The sample is a permalloy square exhibiting a Landau domain structure with a vertex core in the middle. Fig. $5 \mathbf{b}$ illustrates how the vertex core motion forms a vertexcore spin orbit. When a vortex core is displaced from the center of a ferromagnetic disc, the demagnetization energy serves as a restorative force, and the gyrotropic and precessional dynamics can be captured using a harmonic oscillator model. As a result of the core 
displacement, magnetic 'charges' form on the edges of the disc. These weakly coupled magnetostatic interactions are long range but have a small interaction cross section and even edge overlapped direct exchange discs may demonstrate complex non-linear modes. Alternatively a stacked geometry increases the magnetic interaction over the entire surface areas of the ferromagnetic layers leading to stronger coupling of neighboring vortices.

Fig. 5 c-f show Lorentz micrographs of the vortex core precession orbit at various driving frequencies. The current was applied along the horizontal direction (as marked in Fig. 5a), with the current density maintained at $7.7 \times 10^{10} \mathrm{~A} / \mathrm{m}^{2}$. As the vortex precesses about the central equilibrium point, the domain walls of the Landau structure oscillate about their equilibrium positions, creating blurred domain contrast at their boundaries. The domains, which are pinned by each of the four corners of the spin structure and the vortex core, move with the vortex core. Imaging of the gyrotropic motion reveals subtle changes in the ellipticity, amplitude and tilt of the orbit as the vortex is driven through resonance, providing a robust method to determine the adiabatic, non-adiabatic spin torque parameters with unprecedented precision. Fig. 5 c-e show the change of vortex-core orbit size of the counter clockwise (CCW) domains, from the remanence (c), off resonance $(\mathrm{d}, 200 \mathrm{MHz})$ and at resonance $(\mathrm{e}, 180 \mathrm{MHz})$ state, indicating the core reaches maximum at resonance with rather circular orbit. In contrast, as the frequency moves off resonance, the ellipticity of the orbit, which follows an almost linear relationship with the normalized frequency, is clearly visible as illustrated in Fig.2(d) and (f) for vortices of (polarity $=+1$, CCW) and (polarity $=+1$, CW). Fig. 5g depicts the measured tilt from the current direction versus frequency for the same element with a theoretical prediction superimposed (solid line). The measurements of the orbit amplitudes for both chiralities and as a function of frequency allow for the unambiguous determination of the non-adiabatic parameter $\beta$ and the viscous damping parameter $\alpha$, respectively. The orbital tilts are sensitive to the relative magnitudes of all three driving contributions: adiabatic, non-adiabatic, and Oersted field contribution. Hence, mapping the tilt angle with respect to frequency allows additional determination of the degree of spin polarization, $\mathrm{P}$, though in practice, local pinning effects can influence low amplitude oscillations and may complicate the measurement of spin polarization.

Another example is direct imaging of mode-coupled magnetic vortices. Interlayer coupled vortices where the magnetic interaction was altered in asymmetric disc heterostructures of 
Permalloy/Copper/Permalloy Py/Cu/Py). By reducing the non-magnetic spacer layer thicknesses of copper, indirect exchange interactions strongly coupled vortices of non-overlapping eigenmodes, creating spatially coherent core oscillations with a singular peak in the subgigahertz frequency spectrum corresponding to a mode-coupled gyrotropic motion. This is in contrast to the magnetostatic coupled systems where the cores interact in a chaotic manner and the characteristic periodic motion cannot be detected. The coherent oscillations found in the indirect exchange coupled disc heterostructures serves as a basis for more complex coupled systems and could be used as a means of amplification and tuning of the eigenmodes in strongly interacting multi-vortex systems. Fig. 5 h shows a schematic diagram of the heterostructures, where the thickness of the two ferromagnetic layers where fixed at $25 \mathrm{~nm}$ and $15 \mathrm{~nm}$ of Py, separated by a thin $\mathrm{Cu}$ layer. At equilibrium, the vortex cores reside in the center of the disc at the energy minimum of the potential landscape. If the two ferromagnetic layers of the device are isolated and excited with an RF field at resonance, the orbital amplitude of the steady state vortex core-path trace $(\mathrm{r} 1, \mathrm{r} 2)$ as well as the eigenfrequency $(\omega 1, \omega 2)$ would be distinct as shown in Fig. 5i. The two vortices can be interpreted as two harmonic oscillators with dissimilar restorative spring constants and masses due to the thickness asymmetry. The non-magnetic $\mathrm{Cu}$ layer serves as a means of modifying the interlayer coupling strength. The asymmetry also provides unique behavior as non-overlapping eigenmodes should become evident in the frequency spectrum and can also be used to independently control the states of the vortices. The coherent oscillations found in the indirect exchange coupled disc heterostructures serves as a basis for more complex coupled systems and could be used as a means of amplification and tuning of the eigenmodes in strongly interacting multi-vortex systems. It provides critical insights of the fundamental features of collective vortex-based microwave generators, such as their steady-state amplitudes, tenability and mode-coupled gyrotropic vortex motion. The ability to image coupled multiple vortex cores and excite them into resonant modes has implications for fundamental dynamics of interacting topological defects as well as novel technological utilities, such as magnetic nano oscillators and antennae.

\section{Outlook}

In summary, in situ electron microscopy is a fascinating area of research which covers of a wide range of topics and attracts abroad interest. In this article, it was demonstrated that the development of liquid environmental cells allows imaging of materials transformations in liquids 
with high spatial and temporal resolution and significant discoveries have been made in materials chemistry. Exciting results and progress have also been achieved in the understanding of ferroelectric and ferromagnetic vortex with the sample stage advancement. In both cases, the in situ TEM observation benefit from the aberration corrected electron microscopy with high spatial resolution and other advanced electron microscopy imaging and spectroscopic techniques.

For liquid cell experiments, when electron beam penetrates through a thick sample including two SiNx nitride membranes and liquid film, limited resolution of TEM imaging can be achieved. However, atomic resolution has been obtained using liquid cells with ultra SiNx membranes ( 10nm) and much thinner liquid layer ( less than $50 \mathrm{~nm}$ ). Especially, the graphene liquid cells may take the full advantages of aberration corrected TEM. Imaging under TEM mode allows viewing dynamic processes of materials in real time by integrating fast electron detection technology. However, scanning (S)TEM offer unique advantages of imaging through a thicker layer of liquid, although temporal resolution of imaging could be compromised. In the future, breakthroughs in science and technologies on imaging materials dynamics in liquids with improved resolution, control of environments and electron beam effects are yet to come. There is no doubt that liquid cell TEM technologies will experience revolutionary growth in the near future. Improving the spatial resolution and signal-to-noise ratio are the key for future development of liquid cell TEM. So far, the study of electrochemical processes in a liquid cell is limited to low resolution imaging, which is partly constrained electron beam damage. Therefore, controlling of electron beam damage is crucial. In addition, liquid cells with well calibrated environment, precursor mixing, controlled applied stimuli, etc. are often required for the study of certain reactions.

In general, improving the temporal resolution is significant a variety of in situ TEM studies of transformation dynamics, including molecular dynamics in liquids, nucleation events, ferroelectric or ferromagnetic domain switching, dislocations propagation under mechanical straining, etc. With the newly developed fast direct electron detector, imaging with a frame rate of 1600 frames per second has been achieved already. Future improvement of the electron detection technologies, i.e., in $\mu \mathrm{s}$, ns, ps or fs regime, would allow for exploring molecular, atomic events or different phonon modes or transitions.

Lastly, with continuing advances in nanofabrication technology and fine control of sample stages, more functions and better control of in situ experiment will be achieved. With the 
aberration corrected TEM, fast electron detection and better sample environment control, fast growth and broader impact of in situ electron microscopy are expected.

Acknowledgements: H. Z. thanks Hong-Gang Liao, Zhiyuan Zeng and Kaiyang Niu for their research on liquid cell TEM. She would also like to thank the funding support from U.S. DOEBES, Materials Science and Engineering Division. Y.Z. would like to thank Shaobo Cheng, Jun Li, and Myung-Geun Han for their research on ferroelectric vortices and Shawn Pollard and Javier Pulecio on ferromagnetic vortices. The work at Brookhaven National Lab was supported by the U.S. DOE-BES, Materials Science and Engineering Division, under Contract No. DESC0012704.

\section{References:}

1. Lopez-Acevedo, O., et al., Quantum size effects in ambient CO oxidation catalysed by ligandprotected gold clusters. Nature Chemistry, 2010. 2(4): p. 329-334.

2. Zheng, H.M., Y.S. Meng, and Y.M. Zhu, Frontiers of in situ electron microscopy. Mrs Bulletin, 2015. 40(1): p. 12-18.

3. Ross, F.M., Opportunities and challenges in liquid cell electron microscopy. Science (New York, N.Y.), 2015. 350(6267): p. aaa9886-aaa9886.

4. Liao, H.G. and H.M. Zheng, Liquid Cell Transmission Electron Microscopy. Annual Review of Physical Chemistry, 2016. 66.

5. de Jonge, N., M. Pfaff, and D.B. Peckys, Practical Aspects of Transmission Electron Microscopy in Liquid, in Advances in Imaging and Electron Physics, Vol 186, P.W. Hawkes, Editor 2014, Elsevier Academic Press Inc: San Diego. p. 1-37.

6. Ross, F.M., Opportunities and challenges in liquid cell electron microscopy. Science, 2015. 350(6267).

7. Liao, H.G. and H.M. Zheng, Liquid Cell Transmission Electron Microscopy, in Annual Review of Physical Chemistry, Vol 67, M.A. Johnson and T.J. Martinez, Editors. 2016, Annual Reviews: Palo Alto. p. 719-+.

8. Zheng, H.M., et al., Observation of Single Colloidal Platinum Nanocrystal Growth Trajectories. Science, 2009. 324(5932): p. 1309-1312.

9. Yurtsever, A., R.M. van der Veen, and A.H. Zewail, Subparticle Ultrafast Spectrum Imaging in 4D Electron Microscopy. Science, 2012. 335(6064): p. 59-64.

10. Yurtsever, A. and A.H. Zewail, 4D Nanoscale Diffraction Observed by Convergent-Beam Ultrafast Electron Microscopy. Science, 2009. 326(5953): p. 708-712.

11. Vanacore, G.M., A.W.P. Fitzpatrick, and A.H. Zewail, Four-dimensional electron microscopy: Ultrafast imaging, diffraction and spectroscopy in materials science and biology. Nano Today, 2016. 11(2): p. 228-249.

12. Yu, Q., et al., Origin of dramatic oxygen solute strengthening effect in titanium. Science, 2015. 347(6222): p. 635-639.

13. Shan, Z.W., et al., Mechanical annealing and source-limited deformation in submicrometrediameter Ni crystals. Nature Materials, 2008. 7(2): p. 115-119.

14. Nelson, C.T., et al., Domain Dynamics During Ferroelectric Switching. Science, 2011. 334(6058): p. 968-971. 
15. Li, L.Z., J.R. Jokisaari, and X.Q. Pan, In situ electron microscopy of ferroelectric domains. Mrs Bulletin, 2015. 40(1): p. 53-61.

16. Bunkov, Y.M. and H. Godfrin, Topological defects and the non-equilibrium dynamics of symmetry breaking phase transitions. Vol. 549. 2012: Springer Science \& Business Media.

17. Ovid'Ko, I. and A. Romanov, Methods of topological obstruction theory in condensed matter physics. Communications in mathematical physics, 1986. 105(3): p. 443-453.

18. Kibble, T.W., Topology of cosmic domains and strings. Journal of Physics A: Mathematical and General, 1976. 9(8): p. 1387.

19. Mermin, N.D., The topological theory of defects in ordered media. Reviews of Modern Physics, 1979. 51(3): p. 591.

20. Trebin, H.-R., The topology of non-uniform media in condensed matter physics. Advances in Physics, 1982. 31(3): p. 195-254.

21. Vilenkin, A. and E.P.S. Shellard, Cosmic strings and other topological defects2000: Cambridge University Press.

22. Yu, X., et al., Real-space observation of a two-dimensional skyrmion crystal. Nature, 2010. 465(7300): p. 901-904.

23. Das, H., et al., Bulk magnetoelectricity in the hexagonal manganites and ferrites. Nature Communications, 2014. 5.

24. Meier, D., et al., Anisotropic conductance at improper ferroelectric domain walls. Nature Materials, 2012. 11(4): p. 284-288.

25. Wu, W., et al., Conduction of topologically protected charged ferroelectric domain walls. Physical Review Letters, 2012. 108(7): p. 077203.

26. Lavrentovich, O., Topological defects in dispersed words and worlds around liquid crystals, or liquid crystal drops. Liquid crystals, 1998. 24(1): p. 117-126.

27. Fiebig, M., et al., Observation of coupled magnetic and electric domains. Nature, 2002. 419(6909): p. 818-820.

28. Huang, F.-T., et al., Duality of topological defects in hexagonal manganites. Physical Review Letters, 2014. 113(26): p. 267602.

29. de Jonge, N. and F.M. Ross, Electron microscopy of specimens in liquid. Nat Nano, 2011. 6(11): p. 695-704.

30. Marton, L., La microscopie electronique des objets biologiques. Acad Roy Belgique Bull Cl Sci, 1934. 20-133(5): p. 439-911.

31. Becker, J., O. Schubert, and C. Sonnichsen, Gold nanoparticle growth monitored in situ using a novel fast optical single-particle spectroscopy method. Nano letters, 2007. 7(6): p. 1664-1669.

32. Sun, Y. and Y. Ren, In Situ Synchrotron X-Ray Techniques for Real-Time Probing of Colloidal Nanoparticle Synthesis. Particle \& Particle Systems Characterization, 2013. 30(5): p. 399-419.

33. Steinfeldt, N., In Situ Monitoring of Pt Nanoparticle Formation in Ethylene Glycol Solution by SAXS-Influence of the NaOH to Pt Ratio. Langmuir, 2012. 28(36): p. 13072-13079.

34. Polte, J., et al., Nucleation and Growth of Gold Nanoparticles Studied via in situ Small Angle X-ray Scattering at Millisecond Time Resolution. ACS nano, 2010. 4(2): p. 1076-1082.

35. Abecassis, B., et al., Probing in situ the nucleation and growth of gold nanoparticles by smallangle $x$-ray scattering. Nano letters, 2007. 7(6): p. 1723-1727.

36. Miao, J., et al., Three-dimensional GaN-Ga2O3 core shell structure revealed by X-ray diffraction microscopy. Physical Review Letters, 2006. 97(21): p. 215503.

37. Ramesh, G.V., B. Sreedhar, and T.P. Radhakrishnan, Real time monitoring of the in situ growth of silver nanoparticles in a polymer film under ambient conditions. Physical Chemistry Chemical Physics, 2009. 11(43): p. 10059-10063. 
38. Simm, A.O., et al., AFM studies of metal deposition: Instantaneous nucleation and the growth of cobalt nanoparticles on boron-doped diamond electrodes. Chemphyschem, 2006. 7(3): p. 704709.

39. Kolmakov, A. and D.W. Goodman, In situ scanning tunneling microscopy of oxide-supported metal clusters: Nucleation, growth, and thermal evolution of individual particles. Chemical Record, 2002. 2(6): p. 446-457.

40. Liao, H.G., et al., Real-Time Imaging of Pt3Fe Nanorod Growth in Solution. Science, 2012. 336(6084): p. 1011-1014.

41. Gibbs, J.W., et al., The collected works of J. Willard Gibbs1902: Longmans, Green and Co.

42. Wulff, G., On the question of speed of growth and dissolution of crystal surfaces. Zeitschrift Fur Krystallographie Und Mineralogie, 1901. 34(5/6): p. 449-530.

43. Xia, Y., et al., Shape-Controlled Synthesis of Metal Nanocrystals: Simple Chemistry Meets Complex Physics? Angewandte Chemie-International Edition, 2009. 48(1): p. 60-103.

44. Tian, N., et al., Synthesis of tetrahexahedral platinum nanocrystals with high-index facets and high electro-oxidation activity. Science, 2007. 316(5825): p. 732-735.

45. Bealing, C.R., et al., Predicting Nanocrystal Shape through Consideration of Surface-Ligand Interactions. ACS nano, 2012. 6(3): p. 2118-2127.

46. Ringe, E., R.P. Van Duyne, and L.D. Marks, Wulff Construction for Alloy Nanoparticles. Nano letters, 2011. 11(8): p. 3399-3403.

47. Liao, H.G., et al., Facet Development during Platinum Nanocube Growth Science, 2014. 345(6199): p. 916-919.

48. Park, J., et al., 3D structure of individual nanocrystals in solution by electron microscopy. Science, 2015. 349(6245): p. 290-295.

49. Yuk, J.M., et al., High-Resolution EM of Colloidal Nanocrystal Growth Using Graphene Liquid Cells. Science, 2012. 336(6077): p. 61-64.

50. Stephenson, T., et al., Lithium ion battery applications of molybdenum disulfide (MoS2) nanocomposites. Energy \& Environmental Science, 2014. 7(1): p. 209-231.

51. RadisavljevicB, et al., Single-layer MoS2 transistors. Nat Nano, 2011. 6(3): p. 147-150.

52. Zeng, Z., et al., Single-Layer Semiconducting Nanosheets: High-Yield Preparation and Device Fabrication. Angewandte Chemie International Edition, 2011. 50(47): p. 11093-11097.

53. Zeng, Z., et al., An Effective Method for the Fabrication of Few-Layer-Thick Inorganic Nanosheets. Angewandte Chemie International Edition, 2012. 51(36): p. 9052-9056.

54. Fang, X.P., et al., Mechanism of Lithium Storage in MoS2 and the Feasibility of Using Li2S/Mo Nanocomposites as Cathode Materials for Lithium-Sulfur Batteries. Chemistry-an Asian Journal, 2012. 7(5): p. 1013-1017.

55. Brazovski, S. and N. Kirova, Theory of plastic flows of CDWs in application to a current conversion. Le Journal de Physique IV, 1999. 9(PR10): p. Pr10-139-Pr10-143.

56. Zweck, J., Lorentz Microscopy, in In-Situ Electron Microscopy2012, Wiley-VCH Verlag GmbH \& Co. KGaA. p. 347-369.

57. Huang, L., M.A. Schofield, and Y. Zhu, Control of Double-Vortex Domain Configurations in a Shape-Engineered Trilayer Nanomagnet System. Advanced Materials, 2010. 22(4): p. 492-495.

58. Varón, M., et al., Dipolar magnetism in ordered and disordered low-dimensional nanoparticle assemblies. Scientific reports, 2013. 3: p. 1234.

59. $\mathrm{Yu}, \mathrm{X}$., et al., Biskyrmion states and their current-driven motion in a layered manganite. Nature Communications, 2014. 5.

60. $\mathrm{Yu}, \mathrm{X}$., et al., Skyrmion flow near room temperature in an ultralow current density. Nature Communications, 2012. 3: p. 988. 
61. Budruk, A., et al., In situ Lorentz TEM magnetization studies on a Fe-Pd-Co martensitic alloy. Acta Materialia, 2011. 59(17): p. 6646-6657.

62. Kohn, A., et al., The antiferromagnetic structures of IrMn3 and their influence on exchange-bias. Scientific reports, 2013. 3.

63. Shindo, D., et al., Electron holography of Fe-based nanocrystalline magnetic materials. Journal of applied physics, 2004. 95(11): p. 6521-6526.

64. Park, H., et al., Direct observation of magnetization reversal in thin Nd2Fe14B film. Journal of applied physics, 2005. 97(3): p. 033908.

65. Pollard, S., et al., Direct dynamic imaging of non-adiabatic spin torque effects. Nature Communications, 2012. 3: p. 1028.

66. Pulecio, J., et al., Coherence and modality of driven interlayer-coupled magnetic vortices. Nature Communications, 2014. 5.

67. Choi, T., et al., Insulating interlocked ferroelectric and structural antiphase domain walls in multiferroic YMnO3. Nature Materials, 2010. 9(3): p. 253-258.

68. Li, J., et al., Homotopy-Theoretic Study \& Atomic-Scale Observation of Vortex Domains in Hexagonal Manganites. Scientific reports, 2016. 6.

69. Han, M.G., et al., Ferroelectric switching dynamics of topological vortex domains in a hexagonal manganite. Advanced Materials, 2013. 25(17): p. 2415-2421.

70. Li, J., et al., Ferroelectric annular domains in hexagonal manganites. Physical Review B, 2013. 87(9): p. 094106.

71. Han, M.-G., et al., Interface-induced nonswitchable domains in ferroelectric thin films. Nature Communications, 2014. 5.

72. Hÿtch, M., E. Snoeck, and R. Kilaas, Quantitative measurement of displacement and strain fields from HREM micrographs. Ultramicroscopy, 1998. 74(3): p. 131-146.

73. Polking, M.J., et al., Ferroelectric order in individual nanometre-scale crystals. Nature Materials, 2012. 11(8): p. 700-709. 


\section{Figure Captions:}

Fig. 1. Single nanoparticle growth trajectories studied using liquid cell TEM. (a). (I) A liquid cell design. (II) Sequential images showing platinum nanoparticle growth either by monomer attachment or by nanoparticle coalescence, leading to the same particle size. (III) Tracking particle size evolution in (II). (b). Pt-Fe nanocrystal growth and attachment to form nanowires. Random attachment and recrystalization to form a single crystal nanowire. (c). Facet development during Pt nanocube growth. (d). (left) sequential high resolution TEM images of the growth of (111) facet. A cluster of atoms formed on the facet and it was subsequently transformed into a monolayer. (right) the atomic growth of (110) facet.

Fig. 2. Nanocyrstal dynamics observed in a graphene liquid cell. (a). A graphene liquid cell schematic. (b). Sequential images showing Pt nanocrystal formation with atomic resolution. (c). A Pt nanocrystal from a graphene liquid cell after 3D reconstruction.

Fig. 3. In situ study of charge-discharge induced electrode structural changes in an electrochemical cell. (a). A home-made electrochemical liquid cell with two Ti electrodes Li and MoS2 nanoparticles loaded on each side. (b). Integration of advanced imaging techniques, e.g., 4D STEM. (c). Analysis of Ti electrode with MoS2 nanoparticles after discharge process. (II-V) EDS mapping showing elemental distribution. Inset in (III) is the quantitative analysis of elements after deconvelution. (VI) 4D STEM demonstrate small MoS2 nanoparticles in a seemly smooth area of electrode in (I)

Fig. 4 Ferroelectric vortices. (a) Electrostatic interactions between domain walls around a vortex (black dot)-antivortex (red dot) pair in saturated state under downward applied field. The electrostatic charges associated with the domain walls are marked in red (positive) and blue (negative). When the applied field reverses, the ferroelectric domains with polarization parallel to the field expand while anti-parallel to the field shrink. (b) Dark-field image of a vortex and its associated domains obtained with the 001 reflection in $\mathrm{ErMnO}_{3}$ under applied field of $150 \mathrm{kV} / \mathrm{cm}$, showing domain configuration and different types of domain walls. (c) ADF STEM images from the regions indicated with the green-rectangle in (b). Unit cells for each domain near walls are denoted with blue- and red-rectangles. The two ferroelectric domain walls are 
type-B wallswith unit-cell-shift vector 2/3[120] across each wall. Yellow arrows indicate the polarization direction for each domain. (d) An 8-fold vortex in hexagonal $\mathrm{YMnO}_{3}$ in the remnant state observed by atomic resolution dipole mapping. Such a vortex symmetry is crystallographically forbidden but topologically allowed. The vortex core has two edge dislocations associated with it. The inset on the right is the strain map around dislocations near the core and the one on the left shows the corresponding low mag image of the vortex domains. (e) The torus-like order-parameter space of the topological defect of the vortex revealed by Landau-based numerical calculations.

Fig. 5. Ferromagnetic vortices. (a) The vortex precession measurement scheme with in-situ high-frequency (subGHz) current excitation. (b) Vortex core precession principle. (c-f) Lorentz image of a vortex-core of the Py square Landau structure. (c) Without excitation. The magnetic configuration of the four domains is counter clockwise. (d) Clock-wise (CW) orbit over-, counter CW orbit at-, and CCW orbit over-resonance, showing the evolution of the tilt and orbit amplitude with frequency, and for different chiralities. (g) Orbit tilt vs. frequency. The effects of chirality are subtle, and require high spatial resolution to observe them. (i) Orbit radius vs. current density for both chiralities. The variation can be used to separate Oersted field effects on the orbit, as well as determine the degree of non-adiabaticity associated with spin torques. (g) Measured tilt from the current direction versus frequency for the same element at $7.9 \times 10^{10} \mathrm{~A} / \mathrm{m}^{2}$. The small shift in resonance frequency from that of a current of $1 \times 10^{11} \mathrm{~A} / \mathrm{m}^{2}$ is due to joule heating. (h) A patterned disc heterostructure with a non-magnetic spacer $\mathrm{Cu}$ layer sandwiched between two magnetic permalloy layers on top of a Au waveguide and $\mathrm{Si}_{3} \mathrm{Ni}_{4}$ membrane (not to scale). The tri-layered heterostructure contains two magnetic vortex cores due to the different thickness of the permalloy layer. (i) Lorentz image of the projected in-plane magnetization to capture both vortex cores simultaneously. A schematic of the dissimilar gyrotropic core motion at the respective eigen-frequencies with different radius due to the thickness asymmetry. 
Fig. 1.

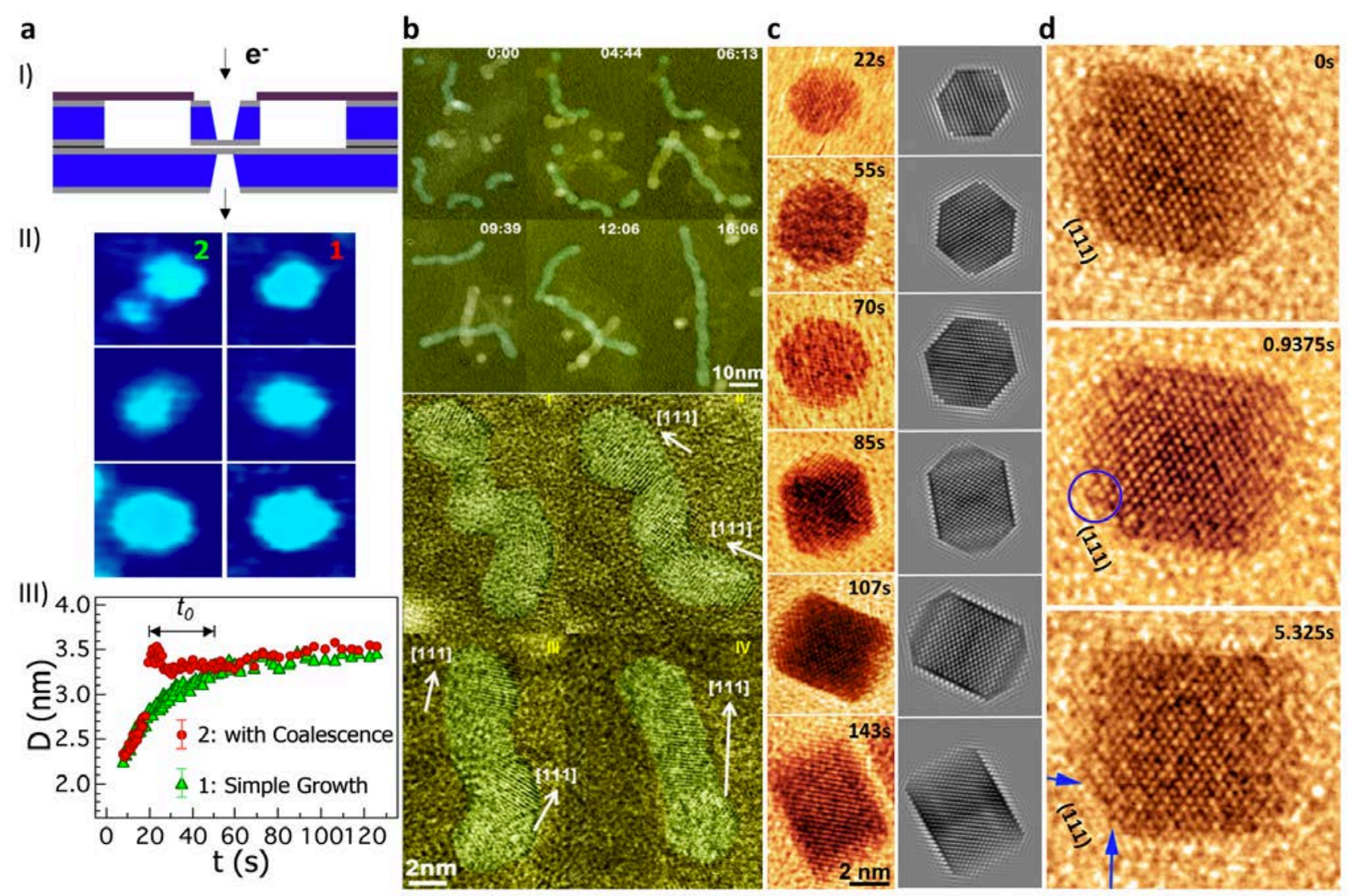


Fig. 2.

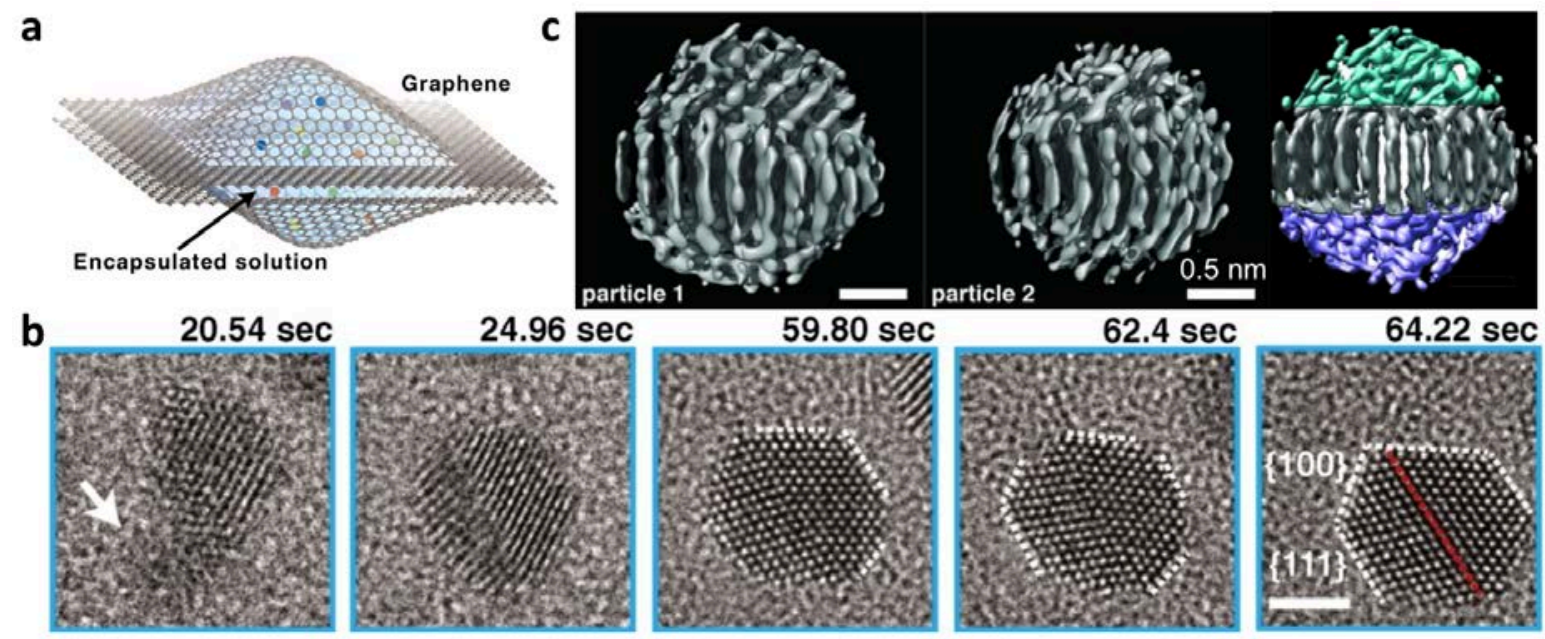


Fig. 3.

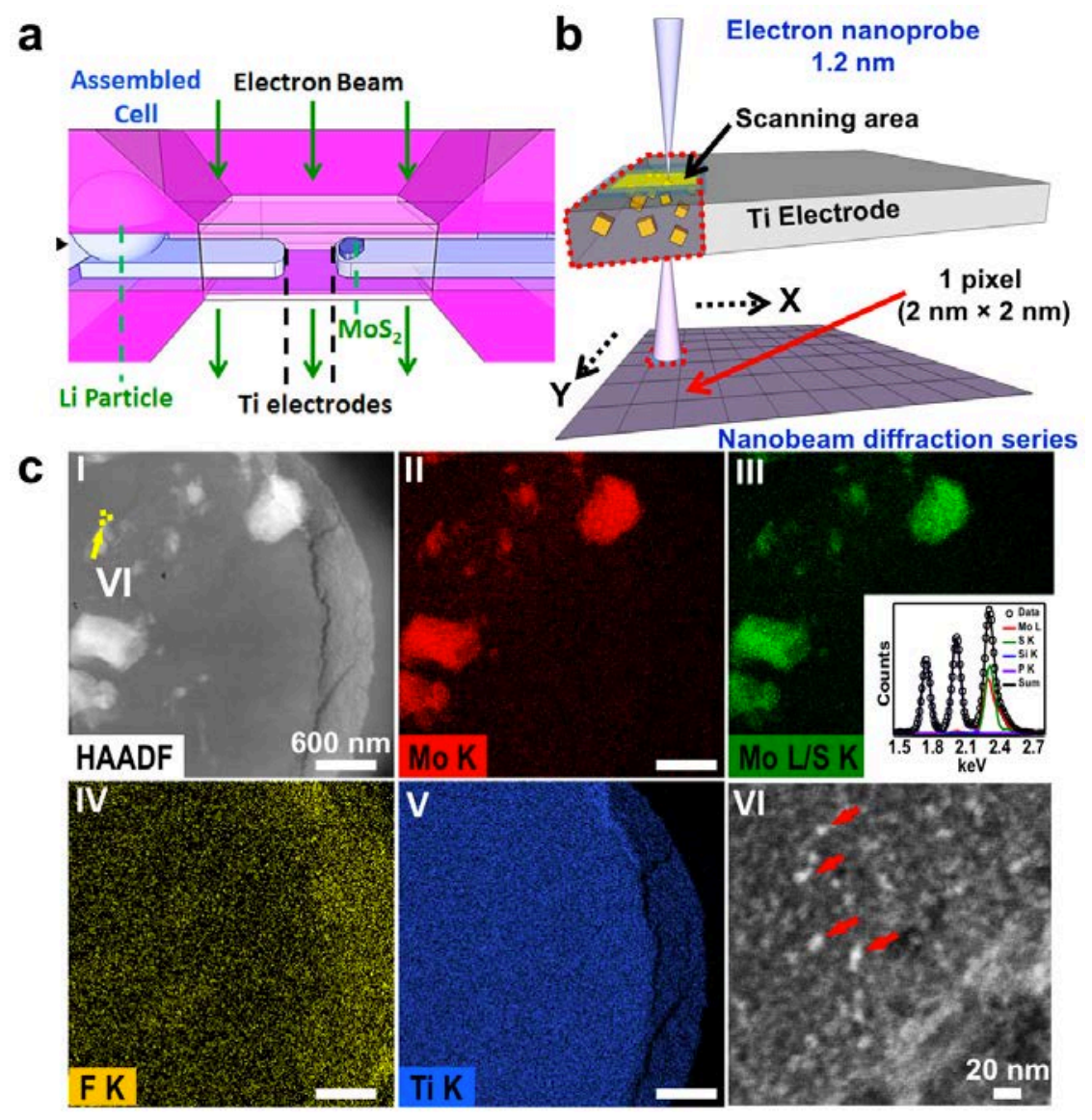


Fig. 4
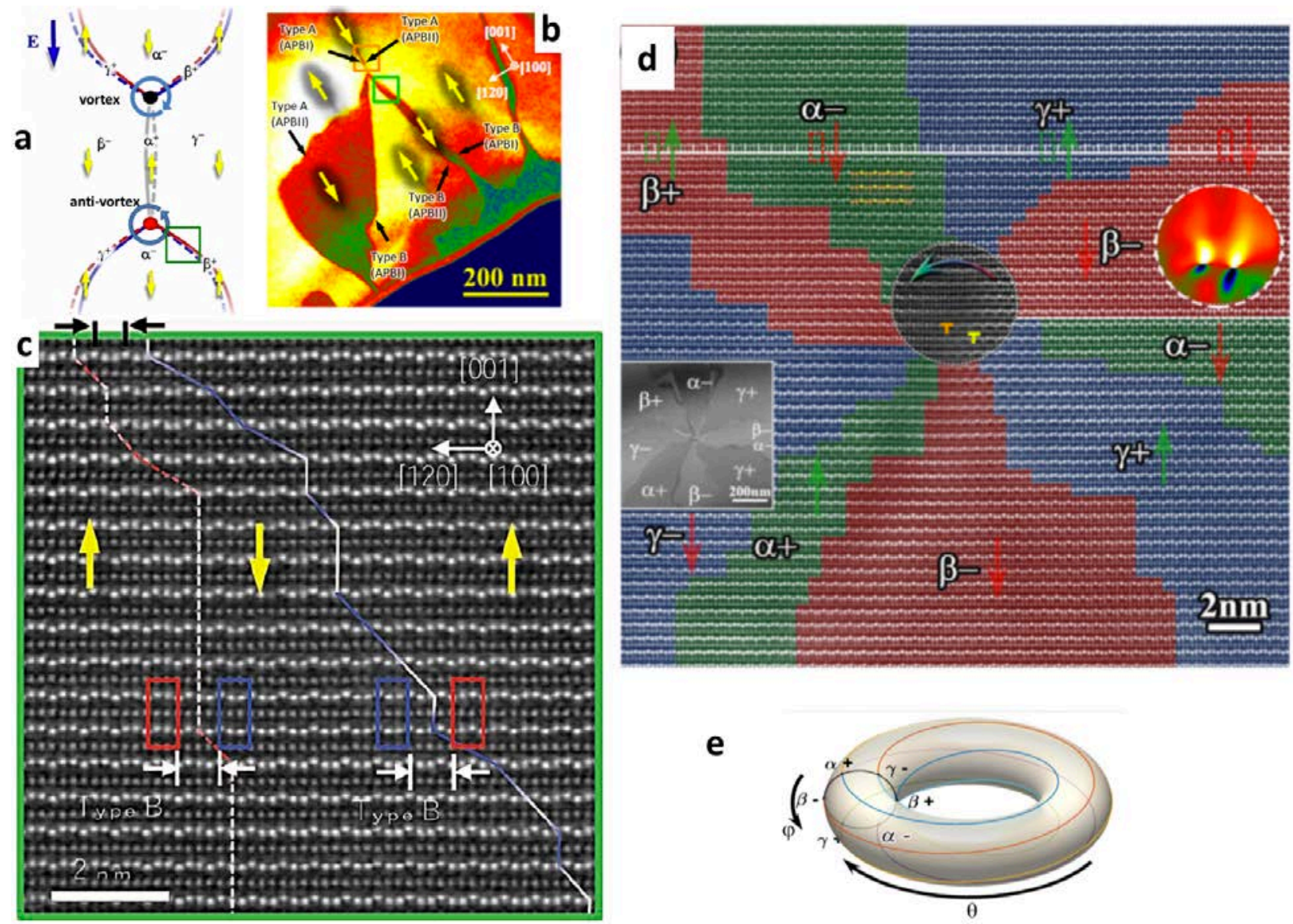

e

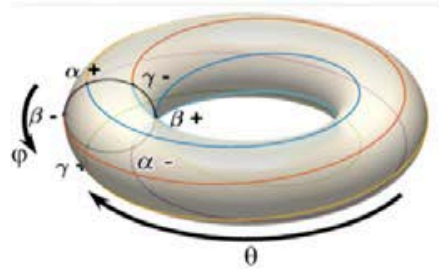


Fig. 5.

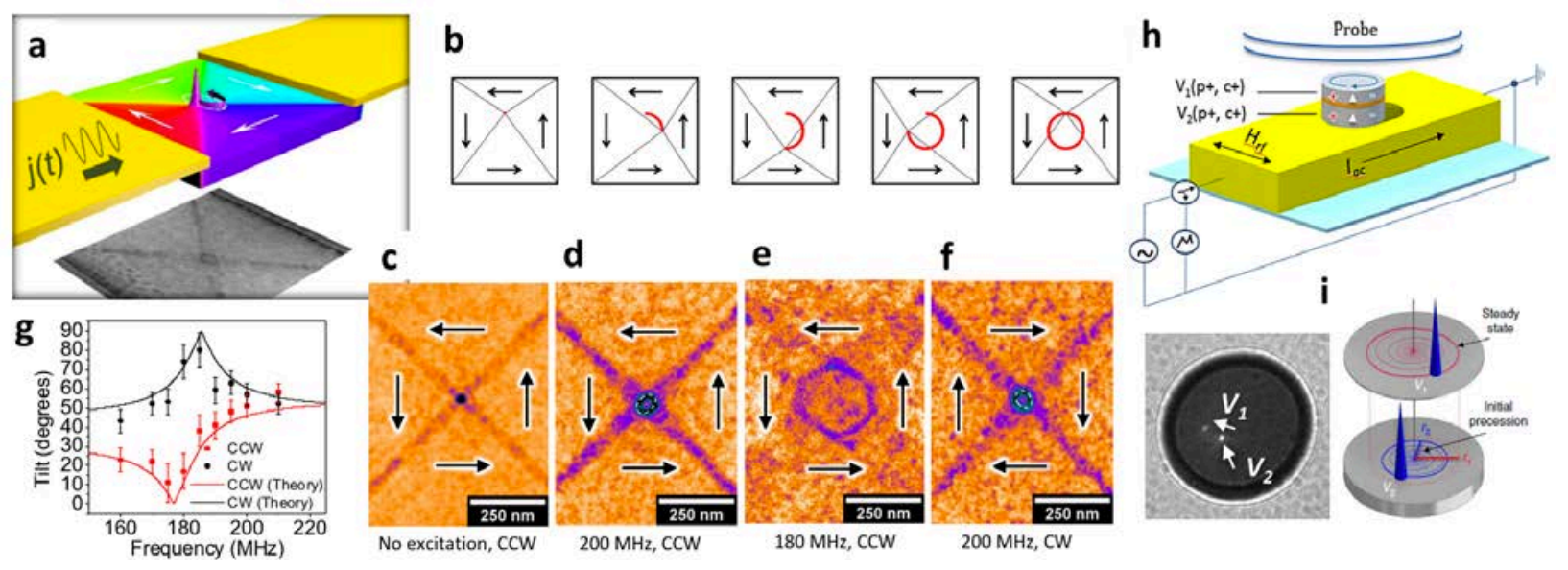

